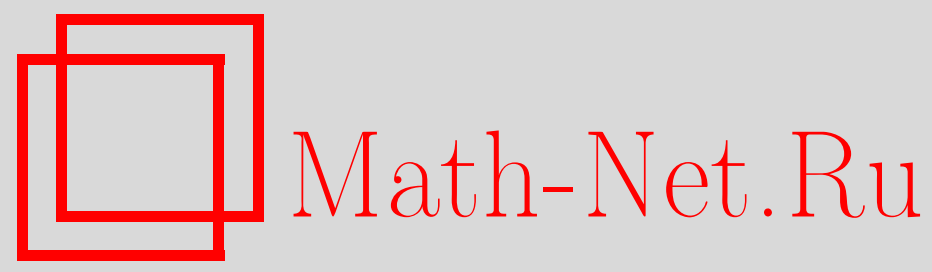

В. С. Кроткин, О. В. Кузьмин, О конструктивном подходе к вычислению мощности классов Райзера, Дискрет. матем., 2009, том 21, выпуск 3, 33-36

DOI: https://doi.org/10.4213/dm1059

Использование Общероссийского математического портала Math-Net.Ru подразумевает, что вы прочитали и согласны с пользовательским соглашением http://www . mathnet.ru/rus/agreement

Параметры загрузки:

IP : 54.92 .164 .108

26 апреля 2023 г., 18:28:12 


\title{
О конструктивном подходе к вычислению мощности классов Райзера
}

\author{
() 2009 г. В. С. Кроткин, О. В. Кузьмин
}

\begin{abstract}
Рассматривается задача вычисления мощности классов квадратных матриц, состоящих из нулей и единиц, с заданными значениями строчных и столбцовых сумм. Получено рекуррентное соотношение, позволяющее вычислять мощности данных классов. Для доказательства используется конструктивный подход - построение комбинаторных объектов с заданными характеристиками и подсчет числа различных объектов. Приводится пример использования полученного соотношения.
\end{abstract}

\section{1. Введение}

Матрица, состоящая из нулей и единиц, называется $(0,1)$-матрицей. Пусть $A=\left(a_{i j}\right)-$ $(0,1)$-матрица размера $m \times n$. Будем следовать обозначениям книги [1]. Пусть $r_{i}-$ сумма единиц в строке $i, s_{j}$ - сумма единиц в столбце $j$, где $i=1, \ldots, m, j=1, \ldots, n$. Пусть $R=\left(r_{1}, \ldots, r_{m}\right)$ и $S=\left(s_{1}, \ldots, s_{n}\right)-$ два вектора, состоящие из значений строчных и столбцовых сумм.

Классом Райзера $\mathfrak{Q}^{n, m}(R, S)$ называется совокупность $(0,1)$-матриц размера $m \times n \mathrm{c}$ заданными векторами $R=\left(r_{1}, \ldots, r_{m}\right)$ и $S=\left(s_{1}, \ldots, s_{n}\right)$ строчных и столбцовых сумм соответственно. С классами Райзера связаны следующие два фундаментальных вопроса.

Когда класс $\mathfrak{U}^{n, m}(R, S)$ непуст?

Чему равно точное число матриц в классе $\mathfrak{2}^{n, m}(R, S)$ ?

Ответом на первый вопрос служат критерии непустоты класса $\mathfrak{P}^{n, m}(R, S)$, например, теорема Гейла-Райзера [1]. Более сложным оказывается второй вопрос о вычислении мощности $\left|\mathfrak{Q}^{n, m}(R, S)\right|$ класса Райзера, так называемая проблема Райзера.

Целочисленные неотрицательные $(0,1)$-матрицы были рассмотрены в конце пятидесятых годов 20-го века в работах Райзера, Фалкерсона и Гейла $[2,3]$ и с тех пор интенсивно изучаются. За полвека изучения проблемы Райзера были получены различные оценки мощности классов $\mathfrak{Q}^{n, m}(R, S)$, а также несколько вычислительных формул для определения точного числа матриц в заданном классе [4].

В нашей работе рассмотрен случай, когда $R=S=(k, \ldots, k), k \in \mathbf{Z}, m=n$. В данном случае класс $\mathfrak{2}^{n, m}(R, S)$ обозначается $\mathfrak{U}^{n}(k)$. Получено рекуррентное соотношение для вычисления $\left|\mathfrak{2}^{n}(k)\right|$. Для доказательства этого соотношения используется конструктивный подход, в соответствии с принципами которого (см. [5]) строится алгоритм построения $(0,1)$-матриц размера $n \times n$, в каждой строке и каждом столбце которых ровно $k$ единиц и $n-k$ нулей и подсчитывается их число. В заключение приводится пример, иллюстрирующий использование предложенного подхода, и дается таблица с результатами вычисления мощностей классов рассматриваемых матриц. 


\section{2. Вычисление мощности классов Райзера}

Следующая теорема дает способ вычисления $\left|\mathfrak{U}^{n}(k)\right|$.

Теорема 1. Число различных квадратных $(0,1)$ матрии порядка $n$, в каждой строке $u$ каждом столбие которых ровно $k$ единиц и $n-k$ нулей, определяется равенством

$$
\left|\mathfrak{U}^{n}(k)\right|=A_{n}(0, \ldots, 0),
$$

где

$$
\begin{aligned}
A_{n}\left(n_{1}, \ldots, n_{k}\right)=\sum_{i_{1}+\ldots+i_{k}=k}\left(\begin{array}{c}
n-n_{1} \\
i_{1}
\end{array}\right)\left(\begin{array}{c}
n_{1}-n_{2} \\
i_{2}
\end{array}\right) & \ldots\left(\begin{array}{c}
n_{k-1}-n_{k} \\
i_{k}
\end{array}\right) \\
& \times A_{n}\left(n_{1}+i_{1}, i_{2}+i_{2}, \ldots, n_{k}+i_{k}\right),
\end{aligned}
$$

суммирование ведется по всем разбиениям $k$ на $k$ цельх неотрицательных слагаемых, $a$ $A_{n}(n, \ldots, n)=1$.

Доказательство. Рассмотрим квадратную матрицу порядка $n$, состоящую только из нулей. Под размещением единицы в матрице будем понимать замену одного из элементов, равных нулю, на единицу. На каждом шаге будем размещать в одной строке данной матрицы по $k$ единиц, учитывая, что в итоге всех размещений в каждом столбце должно оказаться тоже $k$ единиц. Размещения проводим последовательно, начиная с первой строки. Будем нумеровать шаги размещения с 1 до $n$.

Введем следующее обозначение. Пусть $i_{j}^{t}$ - число единиц, размещаемых на шаге $t$, в столбцах, которые уже содержат ровно $j-1$ единиц (не более одной единицы в каждом столбце).

Рассмотрим множество $I=\left\{i_{j}^{t}\right\}, j \in\{2, \ldots, k\}, t \in\{1, \ldots, n\}$.

На первом шаге размещения все столбцы содержат 0 единиц, поэтому $i_{1}^{1}=k$, $i_{2}^{1}=\ldots=t_{k}^{1}=0$.

На втором шаге размещения существуют только столбцы, которые или не содержат единиц, или содержат только одну единицу, размещенную на первом шаге, поэтому $i_{1}^{2}+i_{2}^{2}=k, i_{3}^{2}=\ldots=i_{k}^{2}=0$. На третьем шаге $i_{1}^{3}+i_{2}^{3}+i_{3}^{3}=k, i_{3}^{3}=\ldots=i_{k}^{3}=0$ и так далее. Аналогично на последнем шаге размещения $i_{1}^{n-1}=\ldots=i_{k-1}^{n}=0, i_{k}^{n}=k$, и на предпоследнем $i_{1}^{n-1}=\ldots=i_{k-2}^{n-1}=0, i_{k-1}^{n-1}=\ldots=i_{k}^{n-1}$ и так далее.

После каждого шага размещения в $k$ столбцов добавляется по одной единице.

После первого шага размещения будем иметь $n-i_{1}^{1}=n-k$ столбцов, содержащих 0 единиц, $i_{1}^{1}-i_{2}^{1}=k$ столбцов, содержащих 1 единицу, $i_{2}^{1}-i_{3}^{1}=0$ столбцов, содержащих 2 единицы, и так далее, наконец, $i_{k-1}^{1}-i_{k}^{1}=0$ столбцов, содержащих $k-1$ единиц.

После второго шага размещения будем иметь $n-i_{1}^{1}-i_{1}^{2}=n-k-i_{1}^{2}$ столбцов, содержащих 0 единиц, $i_{1}^{1}-i_{2}^{1}-i_{2}^{1}-i_{2}^{2}=k+i_{1}^{2}-i_{2}^{2}$ столбцов, содержащих 1 единицу, $i_{2}^{1}+i_{2}^{2}-i_{3}^{1}=i_{2}^{2}$ столбцов, содержащих 2 единицы, и так далее, наконец, $i_{k-1}^{1}+i_{k-1}^{2}-i_{k}^{1}-i_{k}^{2}=0$ столбцов, содержащих $k-1$ единиц.

Таким образом, число столбцов, в которых после шага $t-1$ размещено $j-1$ единиц, будет равно $i_{j-1}^{1}+\ldots+i_{j-1}^{t-1}-i_{l}^{1}-\ldots-i_{j}^{t-1}=0, j \in\{2, \ldots, k\}, t \in\{2, \ldots, n\}$.

Введем обозначение $i_{j}^{1}-\ldots-i_{j}^{t-1}=n_{j}^{t}, l \in\{1, \ldots, k\}, t \in\{2, \ldots, n\}$.

Определим множество $N=\left\{n_{j}^{t}\right\}$. Положим $n_{1}^{1}=n_{2}^{1}=\ldots=n_{k}^{1}=0$ и найдем, какие значения могут принимать элементы $n_{j}^{t}, j \in\{1, \ldots, k\}, t \in\{2, \ldots, n\}$. Учитывая 
структуру множества $I=\left\{t_{j}^{t}\right\}$ получаем, что $n_{1}^{2}=k, n_{2}^{2}=\ldots=n+k^{2}=0, n_{1}^{3}=k+i_{1}^{2}$, $n_{2}^{3}=i_{2}^{2}, n_{3}^{4}=\ldots=n_{k}^{3}=0$, и так далее.

Подсчитаем число $A_{n}^{t}\left(n_{1}^{t}, \ldots, n_{k}^{t}\right)$ способов, которыми мы можем разместить $k$ единиц на шаге $t$ :

$$
A_{n}^{t}\left(n_{1}^{t}, \ldots, n_{k}^{t}\right)=\sum_{i_{1}^{t}+\ldots+i_{k}^{t}=k}\left(\begin{array}{c}
n-n_{1}^{t} \\
t_{1}^{t}
\end{array}\right)\left(\begin{array}{c}
n_{1}^{t}-n_{2}^{t} \\
t_{2}^{t}
\end{array}\right) \ldots\left(\begin{array}{c}
n_{k-1}^{t}-n_{k}^{t} \\
t_{k}^{t}
\end{array}\right),
$$

где суммирование ведется по всем разбиениям натурального $k$ на $k$ целых неотрицательных слагаемых.

Зная множество значений, которые могут принимать наборы $\left(n_{1}^{t}, \ldots, n_{k}^{t}\right)$, рассмотрим подробнее процесс размещения единиц на каждом шаге:

$$
\begin{aligned}
A_{n}^{1}\left(n_{1}^{1}, n_{2}^{1}, \ldots, n_{k}^{1}\right)=A_{n}^{1}(0,0, \ldots, 0) & =\sum_{i_{1}^{1}=k}\left(\begin{array}{l}
n \\
i_{1}^{1}
\end{array}\right)=\left(\begin{array}{l}
n \\
k
\end{array}\right), \\
A_{n}^{2}\left(n_{1}^{2}, n_{2}^{2}, \ldots, n_{k}^{2}\right)=A_{n}^{2}(k, 0, \ldots, 0) & =\sum_{i_{1}^{2}+i_{2}^{2}=k}\left(\begin{array}{c}
n-k \\
i_{2}^{1}
\end{array}\right)=\left(\begin{array}{c}
n-k \\
i_{1}^{2}
\end{array}\right)\left(\begin{array}{c}
k \\
i_{2}^{2}
\end{array}\right), \\
A_{n}^{3}\left(n_{1}^{3}, n_{2}^{3}, \ldots, n_{k}^{3}\right)=A_{n}^{3}\left(k+i_{1}^{2}, i_{2}^{2}, \ldots, 0\right) & =\sum_{i_{1}^{3}+i_{2}^{3}+i_{3}^{3}=k}\left(\begin{array}{c}
n-k-i_{1}^{2} \\
i_{1}^{3}
\end{array}\right) k+i_{1}^{2}-i_{2}^{2} i_{2}^{2}\left(\begin{array}{c}
i_{2}^{2} \\
i_{3}^{3}
\end{array}\right)
\end{aligned}
$$

и так далее.

Очевидно, что

$$
\left|\mathfrak{U}^{n}(k)\right|=\prod_{t=1}^{n} A_{n}^{t}\left(n_{1}^{t}, \ldots, n_{k}^{t}\right),
$$

Используя свойства биномиальных коэффициентов и ограничения на их индексы, избавимся от параметра $t$ и запишем данное произведение в виде рекуррентного соотношения, которое соответствует описанному пошаговому процессу размещения и при заданных начальных и конечных условиях дает число всех $n \times n$ матриц, в каждой строке и каждом столбце которых ровно $k$ единиц и $n-k$ нулей,

$$
\begin{aligned}
A_{n}\left(n_{1}, n_{2}, \ldots, n_{k}\right)= & \sum_{i_{1}+\ldots+i_{k}}\left(\begin{array}{c}
n-n_{1} \\
i_{1}
\end{array}\right)\left(\begin{array}{c}
n_{1}-n_{2} \\
i_{2}
\end{array}\right) \\
& \ldots\left(\begin{array}{c}
n_{k-1}-n_{k} \\
i_{k}
\end{array}\right) \\
& \times A_{n}\left(n_{1}=i_{1}, n_{2}+i_{2}, \ldots, n_{k}+i_{k}\right)
\end{aligned}
$$

где суммирование ведется по всем разбиениям $k$ на $k$ целых неотрицательных слагаемых с начальными условиями $n_{1}=n_{2}=\ldots=n+k=0$ и конечными условиями $n_{1}=n_{2}=$ $\ldots=n+k=n$. Теорема доказана.

\section{3. Примеры}

Рассмотрим применение полученного соотношения для вычисления $\left|\mathfrak{U}^{n}(k)\right|$. 
Таблица 1.

\begin{tabular}{|c|l|l|l|l|l|l|}
\hline$n n^{k}$ & 1 & \multicolumn{1}{|c|}{2} & 3 & 4 & 5 & 6 \\
\hline 1 & 1 & & & & & \\
\hline 2 & 2 & 1 & & & & \\
\hline 3 & $3 !$ & $3 !$ & 1 & & & \\
\hline 4 & $4 !$ & 90 & $4 !$ & 1 & & \\
\hline 5 & $5 !$ & 2040 & 2040 & $5 !$ & 1 & \\
\hline 6 & $6 !$ & 67950 & 29720 & 67950 & $6 !$ & 1 \\
\hline
\end{tabular}

Пример 1. Найдем $\left|\mathfrak{U}^{3}(2)\right|=A_{3}(0,0)$. Воспользовавшись теоремой 1 , получаем, что

$$
\begin{aligned}
& A_{3}(0,0)=\sum_{i_{1}+i_{2}=2}\left(\begin{array}{l}
3 \\
i_{1}
\end{array}\right)\left(\begin{array}{l}
0 \\
i_{2}
\end{array}\right) A_{3}\left(0+i_{1}, 0+i_{2}\right)=\left(\begin{array}{l}
3 \\
2
\end{array}\right)\left(\begin{array}{l}
0 \\
0
\end{array}\right) A_{3}(2,0), \\
& A_{3}(2,0)=\sum_{i_{1}+i_{2}=2}\left(\begin{array}{c}
3-2 \\
i_{1}
\end{array}\right)\left(\begin{array}{l}
2 \\
i_{2}
\end{array}\right) A_{3}\left(2+i_{1}, 0+i_{2}\right)=\left(\begin{array}{l}
1 \\
0
\end{array}\right)\left(\begin{array}{l}
2 \\
2
\end{array}\right) A_{3}(2,2)+\left(\begin{array}{l}
1 \\
1
\end{array}\right)\left(\begin{array}{l}
2 \\
1
\end{array}\right) A_{3}(3,1), \\
& A_{3}(2,2)=\sum_{i_{1}+i_{2}=2}\left(\begin{array}{c}
1 \\
i_{1}
\end{array}\right)\left(\begin{array}{l}
0 \\
i_{2}
\end{array}\right) A_{3}\left(2+i_{1}, 2+i_{2}\right)=0, \\
& A_{3}(3,1)=\sum_{i_{1}+i_{2}=2}\left(\begin{array}{c}
0 \\
i_{1}
\end{array}\right)\left(\begin{array}{l}
2 \\
i_{2}
\end{array}\right) A_{3}\left(3+i_{1}, 1+i_{2}\right)=\left(\begin{array}{l}
0 \\
0
\end{array}\right)\left(\begin{array}{l}
2 \\
2
\end{array}\right) A_{3}(3,3)=1 .
\end{aligned}
$$

В итоге получаем, что $\mathfrak{U}^{3}(2)=A_{3}(0,0)=6$.

Таким же образом можно вычислить мощности классов Райзера $\mathfrak{U}^{n}(k)$ при $k, n \in 1, \ldots, 6$, их значения представлены в табл. 1.

\section{Список литературы}

1. Сачков В. Н., Тараканов В. Е., Комбинаторика неотрицательных матрии. ТВП, Москва, 2000.

2. Gale D., A theorem on flows in networks. Pacific J. Math. (1957) 7, 1073-1082.

3. Ryser H. J., Combinatorial properties of matrices of zeros and ones. Canadian J. Math. (1957) 9, 371-377.

4. Wang B. Y., Zhang F., On the precise number of $(0,1)$-matrices in $U(R, S)$. Discrete Math. (1998) 187, 211-220.

5. Марков А. А., О логике конструктивной математики. Знание, Москва, 1972.

Статья поступила 15.04.2009. 